\title{
Stem cell markers in glioma progression and recurrence
}

\author{
KIRSTEN HATTERMANN ${ }^{1 *}$, CHARLOTTE FLÜH ${ }^{2 *}$, \\ DOROTHEE ENGEL ${ }^{1}$, H. MAXIMILIAN MEHDORN ${ }^{2}$, MICHAEL SYNOWITZ ${ }^{2}$, \\ ROLF MENTLEIN $^{1^{*}}$ and JANKA HELD-FEINDT ${ }^{2 *}$ \\ ${ }^{1}$ Department of Anatomy, University of Kiel, D-24098 Kiel; ${ }^{2}$ Department of Neurosurgery, \\ University Medical Center Schleswig-Holstein UKSH, Campus Kiel, D-24105 Kiel, Germany
}

Received March 16, 2016; Accepted June 13, 2016

DOI: 10.3892/ijo.2016.3682

\begin{abstract}
Aggressive cancer cells show histological similarities to embryonic stem cells. As differentiated cells can re-acquire pluripotency and self-renewal by transfection with the transcription factors OCT4, SOX2, KLF4 and MYC, with Nanog as readout for success, we comprehensively investigated their occurrence and frequency in human astrocytomas of different malignancy grades, primary and matched recurrent glioblastomas, short- and long-term glioblastoma cultures and glioma cell lines. Among astrocytomas, mRNA expression of OCT4, MYC and (less robust) KLF4 increased with malignancy, while in recurrent glioblastomas MYC expression slightly decreased. Correlation analysis revealed distinct positive correlation between distinct stem cell markers, and this effect was most prominent in the recurrent glioblastoma cohort. In situ, embryonic stem cell factors were found also in more differentiated tumor regions. Respective cells were rarely actively proliferating and showed single or combined expression signatures, which, at least in parts, corresponded to observed positive correlations of mRNA expression. However, a 'master-marker' defining the complete glioma stem cell subset could not be confirmed. In glioma cell lines, long- and short-term cultures, embryonic markers were detected at comparable levels. Upon exposure to temozolomide, increased expression of KLF4 (and lesser Nanog and OCT4) was observed. Experimental intrinsic overexpression of SOX2,
\end{abstract}

Correspondence to: Dr Kirsten Hattermann, Department of Anatomy, University of Kiel, Olshausenstrasse 40, D-24098 Kiel, Germany

E-mail:k.hattermann@anat.uni-kiel.de

*Contributed equally

Abbreviations: GAPDH, glycerinaldehyde-3-phosphate-dehydrogenase; GBM, glioblastoma multiforme; GFAP, glial fibrillary acidic protein; iPSCs, induced pluripotent stem cells, KLF4, Krüppel-like factor 4; Musashi-1, Musashi (Drosophila) homolog-1; Nanog, 'Tir nan Og'; OCT4, octamer binding transcription factor; qRT-PCR, quantitative reverse transcription-polymerase chain reaction; SOX2, sex determining region Y-box 2; WHO, World Health Organization

Key words: embryonic stem cell marker, glioma stem-like cell, glioma progression, glioma recurrence
KLF4 or OCT4 did not affect the other stem cell factors. The embryonic stem cell factors comprehensively investigated in this project can control self-renewal and pluripotency, and therefore tumorigenicity. They should be considered for the development of future diagnostic and therapeutic strategies.

\section{Introduction}

Gliomas account for approximately $70 \%$ of all human primary brain tumors in adults, with astrocytomas representing the largest group (1). According to increasing malignancy, astrocytomas are classified into pilocytic astrocytomas (WHO grade I) and malignant astrocytomas grade II, III or IV $(2,3)$. Due to its largely heterogeneous phenotype, the most aggressive grade IV tumor, with a patient's median survival time of only 12-15 months, was historically termed glioblastoma multiforme (GBM) (4,5), accompanied by the idea that gliomas may arise from immature precursor cells of the nervous system ('glioblasts').

Following the mayor concepts of the cancer stem cell theory (reviewed for example in ref. 6), the expression, regulation and functional role of stem cell markers has been widely investigated. For gliomas, the expression of stem/progenitor cell markers like CD133, Musashi-1, Nestin, Nanog and PDGFR has been described by us and others $(7,8)$ and reviewed (9). Additionally, glioma cells with stem cell properties have been isolated from tumor samples or established glioma cell lines, based on the expression of stem cell markers (e.g. CD133) or their ability to survive under defined stem cell conditions, and have been investigated in vitro and in vivo concerning their tumor-initiating potential, migratory and proliferative capacity and resistance to chemo- and radiotherapy (reviewed in ref. 10).

In general, the concepts of how cancer stem cells gain their ability to self-renew and proliferate are hardly understood. One reason could be the re-acquisition of embryonic properties enabling pluripotency, which might be comparable to the reprogramming of differentiated somatic cells to induced pluripotent stem cells (iPSCs) by introduction of embryonic stem cell transcription factors. This experimental procedure was firstly described by Takahashi and Yamanaka (11), who introduced 24 candidate factors by retroviral transfection into murine fibroblasts. Based on these candidates, they were able to show, that the combination of four factors, OCT4 (octamer binding transcription factor; synonyms Oct3, POUF5), 
SOX2 (sex determining region Y-box 2), MYC and KLF4 (Krüppel-like factor 4), efficiently induced a reprogramming of the somatic fibroblasts yielding pluripotent iPSCs $(11,12)$. OCT4, MYC, KLF4 and SOX2 as well as the readout for efficient reprogramming, Nanog (Tir nan $\mathrm{Og}$ ), are transcription factors that regulate the expression of a multitude of genes downstream. They are all involved in the maintenance and regulation of stem cells at same and different time-points of development (13-20). Their expression has also been reported in cancer, being predominantly expressed by the cancer stem cell population (21-28).

Nevertheless, whether gliomas originate from a malignantly transformed stem/progenitor cell or if stem cell properties (and marker expression) are regained within the process of malignant transformation of e.g. differentiated glial cells is still unclear.

Thus, to evaluate whether one of the mentioned stem cell markers or transcription factors involved in maintenance and regulation of stem cells could probably be identified as a 'master marker' during glioma progression and recurrence, we investigated their particular transcription in human astrocytomas of different malignancy grades and matched primary and recurrent glioblastoma samples. We also analyzed the correlation between gene expression patterns, showed their intratumoral localization and proliferative state [related to GFAP (glial fibrillary acidic protein) distribution, an astroglial marker, and MIB-1/Ki-67, a proliferation marker], compared their co-expression amongst each other and with the neural stem cell marker Musashi-1 [Musashi homolog-1 (Drosophila)], and analyzed their regulation upon extrinsic chemotherapeutic stress and intrinsic overexpression of SOX2, KLF4 and OCT4 in vitro.

\section{Materials and methods}

Tissue samples and cell cultures. Human astrocytomas of WHO grade I-IV [6 WHO I, 5 WHO II, 5 WHO III, 21 WHO IV (not matched, 14 primary and 7 recurrent)] and matched primary and recurrent glioblastomas (14 different pairs, WHO IV) were surgically dissected tissues from the Department of Neurosurgery (Kiel, Germany). All samples were obtained in accordance with the approved ethical standards of the ethics committee of the University of Kiel and the Helsinki Declaration of 1975 (D 536/15) and after informed consent of the donors. The diagnosis was established by a neuropathologist. Commercial human glioma cell lines were obtained from Deutsches Krebsforschungszentrum (Heidelberg, Germany; U118 and U343) or from the American Type Culture Collection (ATCC; Manassas, VA, USA; A172, T98G and U373). Longterm human primary cultures (A764 and A767) we obtained by dissociation of glioblastoma tissue and repetitive subcultivations, whereas short-term human primary cultures (14/07, 25/07, 76/12 and 96/14) were only used for up to three passages after preparation from tumor tissues. When an adequate amount of tumor tissue was available, matched samples of solid/cultured primary human glioblastomas were used for experiments. All cell lines were cultured in Dulbecco's modified Eagle's medium (DMEM; Invitrogen, Carlsbad, CA, USA) supplemented with $10 \%$ fetal calf serum (FCS). Cells were routinely checked for mycoplasma contamination by PCR.
Quantitative RT-PCR. RNA was isolated using TRIzol reagent (Invitrogen) following the manufacturer's instructions for tissue or cell cultures. DNA was digested by RNaseFree DNase (Fermentas, St. Leon-Rot, Germany; Promega, Madison, WI, USA) and cDNA was synthesized by reverse transcription (Fermentas). Real-time PCR was performed using TaqMan Master Mix and Primer Probes (Applied Biosystems, Foster City, CA, USA): hGAPDH (Hs99999905_ m1), hGFAP (Hs00157674_m1), hOCT4 (Hs00999632_g1), hNanog (Hs02387400_g1), hMusashi-1 (Hs00159291_m1), hSOX2 (Hs00602736_s1), hKLF4 (HS00358836_m1) and hMYC (Hs00153408_m1). Cycle of threshold values $\left(\mathrm{C}_{\mathrm{T}}\right)$ were measured by an ABI 7500 Fast cycler (Applied Biosystems) or the $\mathrm{MyiQ}^{\mathrm{TM}}$ Single Color Real-Time PCR detection system (Bio-Rad Laboratories, Munich, Germany). All genes of interest were normalized to the house-keeping gene glyceraldehyde-3-phosphate dehydrogenase (GAPDH) yielding $\Delta \mathrm{C}_{\mathrm{T}}$ values: $\mathrm{C}_{\mathrm{T}}$ (gene of interest) - $\mathrm{C}_{\mathrm{T}}(\mathrm{GAPDH})$. As $\Delta \mathrm{C}_{\mathrm{T}}$ values mirror the exponential course of the PCR, a $\Delta \mathrm{C}_{\mathrm{T}}$ of 3.33 corresponds to a 10 -fold lower expression compared to GAPDH, a $\Delta \mathrm{C}_{\mathrm{T}}$ value of 6.67 corresponds to a 100 -fold lower expression and so on. For statistical analysis, the relative gene expression compared to GAPDH $\left(2^{-\triangle \mathrm{CT}}\right)$ was employed. To include undetectable samples in mean expressions, these sample values were defined as $21\left(=\Delta \mathrm{C}_{\mathrm{T}}\right.$ that would be obtained after the maximum cycle number). The expression differences between primary and recurrent glioblastomas were calculated as $n$-fold $\Delta \Delta C_{T}$ values $=2^{-\Delta C T \text { primary }-D C T \text { recurrent }}$ and displayed in a grey shade encoded manner. A relative expression value of 1 (equal expression in primary and recurrent tumor) was assigned to $30 \%$ grey. Expression values $>1$ (higher expression in recurrent than primary tumor) were assigned with increasing grey shading, reaching black at 3 -fold (or higher) induction. Expression values $<1$ (indicating lower expression in recurrent than primary glioblastomas) were assigned with lighter grey shadings, increasing to white shading for values reaching 0 . The induction of gene expression upon stimulation is displayed as relative gene expression, $\mathrm{n}$-fold expression changes were calculated as $\Delta \Delta \mathrm{CT}$ values $=$

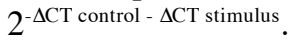

Immunohistochemistry. For immunohistochemistry, $10-\mu \mathrm{m}$ cryo-sections were fixed with an ice-cold acetone-methanol mixture (1:1) for $10 \mathrm{~min}$, blocked for autofluorescence with sudan black (1\% in $70 \%$ ethanol) and for unspecific antibody-binding with $0.5 \%$ glycine $/ 0.5 \%$ bovine serum albumin. Primary antibodies were applied overnight at $4^{\circ} \mathrm{C}$, secondary antibodies were incubated at $37^{\circ} \mathrm{C}$ for $1 \mathrm{~h}$, and nuclei were counterstained with DAPI (Sigma-Aldrich, Hamburg, Germany). Slides were embedded with Thermo Scientific $^{\mathrm{TM}}$ Shandon $^{\mathrm{TM}}$ Immu-Mount ${ }^{\mathrm{TM}}$ (Thermo Shandon, Inc., Pittsburgh, PA, USA). Between each step, slides were washed $3 x$ for 5 min with Tris-buffered saline with $0.1 \%$ Tween (TBS-T). Primary antibodies were anti-GFAP (mouse, 1:100; Dako, Glostrup, Denmark), anti-MIB-1 (Ki-67 clone, mouse, 1:100; Dako), anti-OCT4 (rabbit, 1:200; Cell Signaling Technology, Danvers, MA, USA), anti-SOX2 (rabbit, 1:100; Santa Cruz Biotechnology, Santa Cruz, CA, USA), antiMusashi-1 (1:100, mouse; R\&D Systems, Minneapolis, MN, USA), anti-Nanog (1:500, rabbit; Thermo Fisher Scientific, 

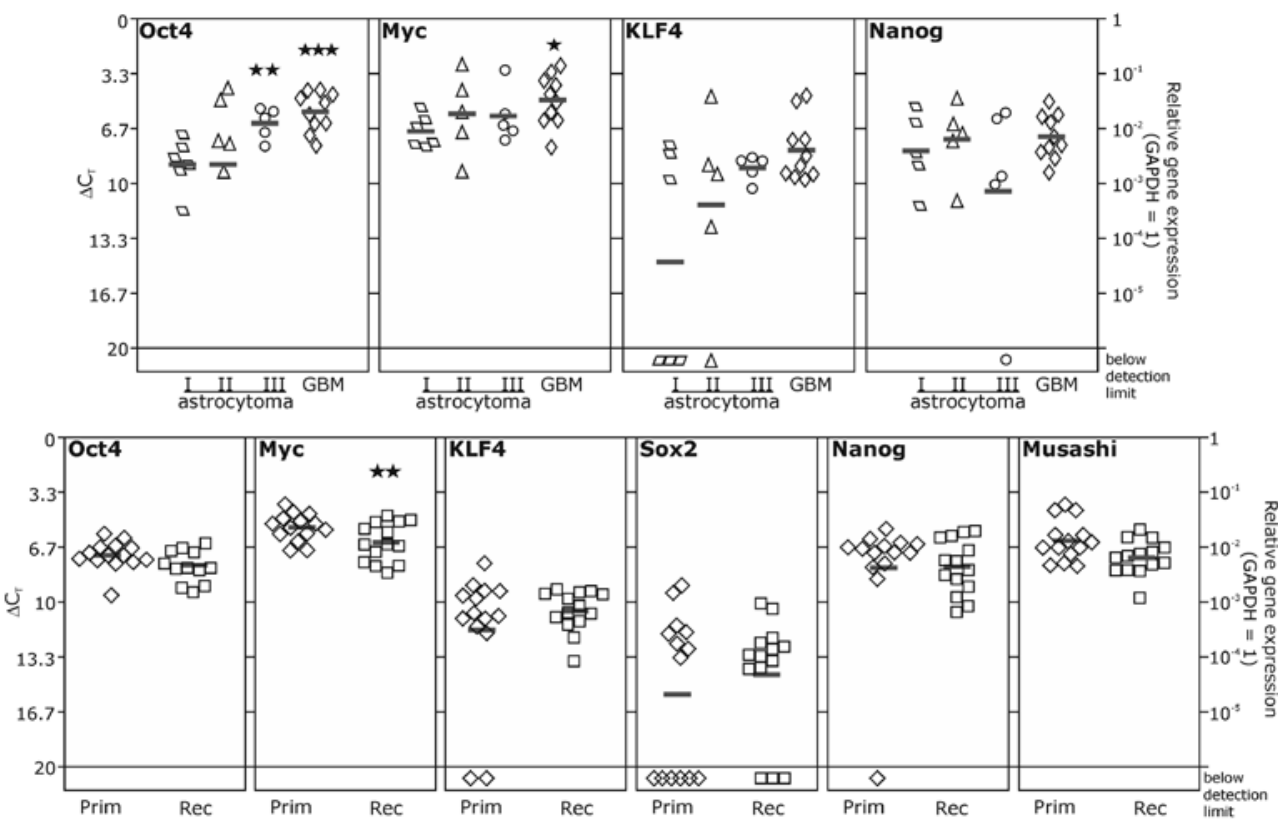

Figure 1. mRNA expression of embryonal stem cell transcription factors in astrocytomas of different malignancy grades and matched primary and recurrent glioblastomas as measured by quantitative RT-PCR. The mRNA expressions of OCT4, Nanog, KLF4 and MYC were detected in astrocytomas of different malignancy grades (according to WHO classification) on moderate to high levels. SOX2 expression was remarkably lower or undetectable (9/28 samples) in matched primary and recurrent glioblastomas (samples derived from the same patient, respectively). In astrocytomas of different malignancy grades, OCT4 and MYC expression increased significantly in highly malignant astrocytomas (in comparison to the benign grade I astrocytomas). An increase of transcription with malignancy was also observed for KLF4. However, due to individual variances, this observation was not statistically significant. In contrast, Nanog was found at about equal levels in astrocytomas of all grades. In matched samples of primary and recurrent glioblastoma samples, the mean expression values for primary and recurrent tumors was stable except for MYC, that was slightly decreased in samples of recurrent tumors. Individual relations between matched primary and recurrent samples are displayed in Table I. For statistical analysis unpaired Student's t-tests for astrocytomas of different malignancy grades were performed and paired Student's t-tests for matched primary and recurrent samples.

Rockford, IL, USA) and anti-KLF4 (1:250, mouse; Pierce/ Thermo Fisher Scientific). When antibodies were derived from different species, they were applied simultaneously and for the antibody controls, both primary antibodies were omitted. If the primary antibodies were derived from the same species, first one primary antibody and its secondary antibody were stained, then unspecific binding was blocked by $\mathrm{F}(\mathrm{ab})$ fragments derived from the very same species [donkey anti-mouse and anti-rabbit $\mathrm{F}(\mathrm{ab})$ fragments, 1:100, from Dianova, Hamburg, Germany] and the second primary and secondary antibodies were applied. In this case, antibody controls were performed by omitting both primary antibodies or, in order to control $\mathrm{F}(\mathrm{ab})$ blocking efficiency, one of them, respectively. Secondary antibodies were donkey anti-mouse or anti-rabbit IgGs labelled with Alexa Fluor 488 or Alexa Fluor 555 (1:800-1:1000; Invitrogen).

Stimulation and transfection experiments. For stimulation and transfection experiments, T98G and A172 glioblastoma cells were seeded subconfluently on 6- or 12-well plates or $75-\mathrm{mm}^{2}$ culture flasks and grown overnight. Media were changed to DMEM $+10 \%$ FCS supplemented with temozolomide or camptothecin (both Sigma-Aldrich) or corresponding concentrations of DMSO as solvent control, cells were incubated for the indicated times and harvested for RNA isolation. For transfection, media were changed to Opti-MEM (Invitrogen), and transfection was performed using Lipofectamine (Invitrogen). The vectors pcDNA3.3eGFP (mock, Addgene plasmid 26822), pcDNA3.3_KLF4 (Addgene plasmid 26815), pcDNA3.3_OCT4 (Addgene plasmid 26816) and
pcDNA3.3_SOX2 (Addgene plasmid 26817) were generated by Derrick Rossi (Harvard University Department of Stem Cell and Regenerative Biology, Boston, MA, USA) (29), and kindly provided by deposition at Addgene, Inc., (Cambridge, MA, USA). Media were changed to DMEM + 10\% FCS $6 \mathrm{~h}$ after transfection, and cells were harvested for RNA isolation after the indicated times.

Statistical analysis. To test significance of expression increase or decrease the Student's t-test was performed. For matched samples (primary-recurrence from the same patient; samples from the same experiment) a paired test was used. Astrocytoma samples of different grades from different patients were analyzed by an unpaired test. For the calculation of mean expression values of tumor samples, undetectable samples were set $\Delta \mathrm{C}_{\mathrm{T}}=21$, as this would commonly represent the detection limit if GAPDH is used as house-keeping gene and 40 amplification cycles are run. For Pearson correlation analysis, expression levels of all investigated stem cell markers of the different astrocytoma malignancy grades, a cohort of mixed primary and recurrent glioblastomas (not matched) and the respective groups of primary and recurrent glioblastomas of matched patient samples were correlated using GraphPad Prism, P-values indicate as follows: " $\mathrm{p}<0.05$, ${ }^{* *} \mathrm{p}<0.01,{ }^{* * *} \mathrm{p}<0.001$ and ${ }^{* * * *} \mathrm{p}<0.0001$.

\section{Results}

Expression of stem cell factors in different malignancy grades of astrocytomas and primary and recurrent GBM. We first investigated the expression of the stem cell factors in 
Table I. Individual expression differences of matched primary and recurrent glioblastomas.

\begin{tabular}{|c|c|c|c|c|c|c|}
\hline & Patients & Oct4 & Nanog & KLF4 & Myc & Sox2 \\
\hline \multirow{15}{*}{ 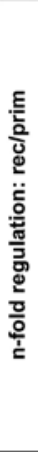 } & \#1 & 2.21 & 1.24 & - & 2.17 & - \\
\hline & $\# 2$ & 1.49 & 2.41 & 5.85 & 1.10 & - \\
\hline & \# 3 & 1.29 & 2.00 & 0.88 & 1.50 & 0.53 \\
\hline & $\# 4$ & 0.83 & 2.00 & 1.30 & 0.36 & - \\
\hline & \# 5 & 1.57 & 0.93 & 2.05 & 0.81 & 0.09 \\
\hline & \#6 & 0.57 & 0.34 & 0.33 & 0.47 & 5.41 \\
\hline & & & & & & \\
\hline & $\# 7$ & 0.51 & 0.41 & 1.23 & 0.61 & - \\
\hline & $\# 8$ & 0.36 & 0.17 & 1.81 & 0.39 & 0.66 \\
\hline & $\# 9$ & 0.60 & 1.28 & 0.36 & 0.19 & 0.37 \\
\hline & $\# 10$ & 1.14 & 0.12 & - & 0.35 & - \\
\hline & \# 11 & 0.11 & 0.33 & 0.94 & 0.34 & - \\
\hline & $\# 12$ & 0.64 & 0.72 & 0.91 & 0.28 & - \\
\hline & $\# 13$ & 0.39 & 0.14 & 0.26 & 0.83 & 0.60 \\
\hline & \# 14 & 0.27 & - & 0.17 & 0.26 & 0.07 \\
\hline
\end{tabular}

The relative mRNA expression change of recurrent glioblastomas in comparison to their matched primary tumors was calculated as n-fold

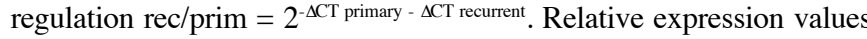
were assigned to grey scale shadings with equal expression in primary and recurrent tumor (corresponding to a relative expression value $=1$ ) representing a 30\% grey shading. Expression values $>1$ (higher expression in recurrent than primary tumor) were assigned with increasing grey shading, reaching black at 3-fold (or higher) induction. Expression values $<1$ (indicating lower expression in recurrent than primary glioblastomas) were assigned with lighter grey shadings, increasing to white shading for values reaching 0 . If the expression of a gene was undetectable for one or both samples, the cells were left empty. Notably, downregulation was more frequently observed than upregulation. The sample cohort could be divided into two groups: one group with clear upregulation ( $>2$-fold) of one or more stem cell factors, and the other group with predominantly downregulation. astrocytomas of WHO grade I-IV, with IV representing the glioblastoma multiforme (GBM), and matched primary and recurrent glioblastomas (Fig. 1). However, as increasing expression levels of SOX2 in astrocytomas with higher malignancy have already been described earlier by us and others $(7,30)$, we restricted our analysis in astrocytomas to OCT4, Nanog, KLF4 and MYC. These were detectable on moderate to high levels in astrocytomas of all malignancy grades. However, for Nanog and KLF4, we found also a lack of expression in distinct samples. Comparable results were obtained for the transcription of Nanog, KLF4 and MYC in matched primary and recurrent glioblastoma samples. In these samples, we also investigated the transcription of SOX2, which was considerably lower, and could not be detected in $9 / 28$ primary/recurrent glioblastoma samples. For OCT4 and MYC we observed a significant increase in mRNA expression in highly malignant astrocytomas (grade III and IV for OCT4 and grade IV for MYC) in comparison to the benign grade I astrocytomas. A trend to higher transcription levels in high grade astrocytomas was also visible for KLF4. However, due to high variances within the groups, these results were not statistically robust. In contrast, Nanog mRNA expression was hardly altered in astrocytomas of different grades in our sample cohort. In matched samples of primary and recurrent glioblastomas mean expression levels were not altered during progression from primary to recurrent glioblastomas except for MYC, which was found slightly decreased in recurrent samples. However, regarding the regulation profile of each patient's primary-recurrent pair, we found distinct patterns of upregulation and downregulation of stem cell markers (Table I, n-fold expression changes
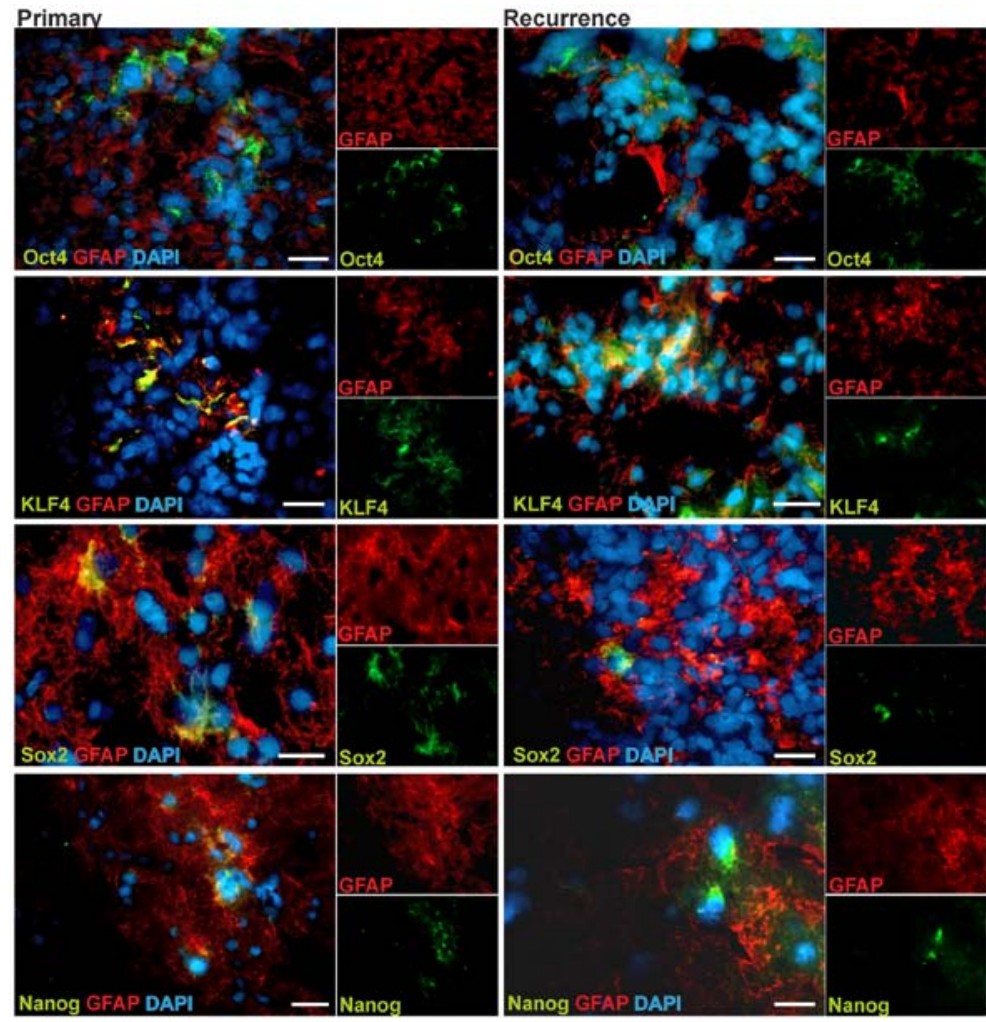

Figure 2. Fluorescence immunostaining of stem cell markers with GFAP in primary and recurrent glioblastomas. Glioblastoma cryo-sections were immunostained for GFAP (red) and stem cell markers OCT4, Nanog, KLF4 and SOX2 (green). Stem cell marker expressing cells were found in small groups or clusters in GFAP-positive tumor regions. Most of these cells showed co-expression of GFAP, although merge of the fluorescence channels was not always observed due to different subcellular localization. However, stem cell marker expressing cells are quite rare ( $\sim 5-10 \%$, compare Table II). Representative staining from 3 primary/recurrent glioblastoma pairs are shown. Bars indicate $20 \mu \mathrm{m}$. 
Table II. Correlation analysis between mRNA expression of stem cell markers and recurrent glioblastomas.

A, Correlation analysis in astrocytomas of different malignancy grades

\begin{tabular}{|c|c|c|c|c|}
\hline \multirow{4}{*}{ Astro I } & $\Sigma 6$ & KLF4 & Oct4 & Nanog \\
\hline & Myc & \multicolumn{2}{|l|}{$0.8742^{\star}$} & $0.9489^{* *}$ \\
\hline & & KLF4 & & \\
\hline & & & Oct4 & \multirow[b]{2}{*}{ Nanog } \\
\hline \multirow{4}{*}{ Astro II } & $\Sigma 5$ & KLF4 & Oct4 & \\
\hline & Myc & & & $0.9125^{*}$ \\
\hline & & KLF4 & & \\
\hline & & & Oct4 & \\
\hline \multirow{4}{*}{ Astro III } & $\Sigma 5$ & KLF4 & \multicolumn{2}{|r|}{ Nanog } \\
\hline & Myc & & & \\
\hline & & KLF4 & & \\
\hline & & & Oct4 & \\
\hline \multirow{4}{*}{ GBM IV } & $\Sigma 10$ & KLF4 & \multicolumn{2}{|r|}{ Nanog } \\
\hline & Myc & \multicolumn{3}{|l|}{$0.8686^{* \star *}$} \\
\hline & & KLF4 & & \\
\hline & & & Oct4 & \\
\hline
\end{tabular}

$\mathrm{B}$, Correlation analysis in primary and recurrent glioblastomas

\begin{tabular}{|c|c|c|c|c|c|}
\hline \multirow{5}{*}{ Primary } & $\Sigma 14$ & KLF4 & Oct4 & Nanog & Sox2 \\
\hline & Myc & \multirow{4}{*}{ KLF4 } & & & \\
\hline & & & \multicolumn{3}{|l|}{$0.7022^{* \star}$} \\
\hline & & & \multirow[t]{2}{*}{ Oct4 } & & \\
\hline & & & & Nanog & \\
\hline \multirow{5}{*}{ Recurrent } & $\sum 14$ & KLF4 & Oct4 & Nanog & Sox2 \\
\hline & Myc & & $0.7588^{* \star}$ & $0.6836^{\star \star \star}$ & \\
\hline & & KLF4 & $0.6530^{*}$ & $0.7343^{* \star}$ & \\
\hline & & & Oct4 & $0.8717^{* \ldots \ldots k}$ & \\
\hline & & & & Nanog & \\
\hline
\end{tabular}

In different astrocytoma grades distinct correlations were observed, especially in combinations with MYC. While in the primary glioblastoma cohort only a positive correlation between KLF4 and OCT4 was detected, in recurrences frequent correlations occurred between several markers. Pearson correlation analysis was performed for each stem cell marker combination, but for clarity, only significant correlations were displayed together with asterisks indicating their P-value $\left({ }^{*} \mathrm{P}<0.05,{ }^{* *} \mathrm{P}<0.01,{ }^{* * *} \mathrm{P}<0.001,{ }^{* * * *} \mathrm{P}<0.0001\right)$.

between individual primary-recurrent pairs, which are numbered consecutively). Based on these observations, we distinguished one group of primary/recurrent pairs with one or more markers being clearly upregulated ( $>2$-fold; Table I, upper part, pairs 1-6) from a second group showing no clear upregulation, or even downregulation of one or more stem cell markers in the recurrent tumor (Table I, lower part, pairs 7-14).

Interestingly, when analyzing a possible correlation of mRNA expression of individual investigated stem cell markers in both astrocytomas of WHO grade I-IV and matched primary and recurrent glioblastomas (Table II), significant positive correlations were restricted to individual marker combinations for most grades and the primary glioblastomas of the primary/recurrent pairs. In detail, astrocytomas grade I showed significant correlations between $M Y C$ and Nanog as well as MYC and KLF4 expression. In astrocytomas grade II we also observed a significant positive correlation between MYC and Nanog, whereas in astrocytomas grade III there was no correlation between the markers. In astrocytomas grade IV (glioblastomas) a significant positive correlation between KLF4 and MYC expression was detectable (Table IIA). Thus, we could not observe a specific correlation pattern consistent to all astrocytoma malignancy grades. However, one has to keep in mind that the sample groups of astrocytomas grade I-III are quite small due to the limited availability of tumor tissue. Our further analysis of the cohort of matched primary/recurrent glioblastoma pairs revealed in the primary samples only a significant correlation between OCT4 and KLF4 expression, but remarkably, in recurrences several stem cell markers showed statistically significant correlations between their mRNA expression levels (e.g. OCT4-MYC, Nanog-MYC and Nanog-OCT4; Table IIB).

Taken together, embryonal and neural stem cell markers are expressed on mRNA level in astrocytomas of different WHO grades, and primary and recurrent glioblastomas. While OCT4 and MYC expression was elevated with increasing malignancy of astrocytomas, MYC expression was slightly reduced in recurrent tumors compared to the corresponding primary tumor. In addition, a correlation analysis of individual stem cell marker mRNA expression revealed sporadic significant positive correlations between Nanog-MYC or KLF4-MYC expression in glioma progression. Remarkably, significant positive correlations between Nanog or OCT4 with other embryonic stem cell factors were frequently found in glioma recurrence, potentially identifying these markers as highly important in glioma progression and recurrence. Apart from this, the regulation pattern of the matched patient samples revealed that upregulation or downregulation of stem cell markers in the progress of primary to recurrent glioblastoma occurs in an individual, probably patient-specific manner.

In situ expression and localization of stem cell factors. To investigate the localization and regional distribution and their potential alteration in glioblastoma progression, we prepared fluorescence stainings of OCT4, SOX2, KLF4 and Nanog using 3 pairs of matched primary and recurrent glioblastoma samples. The protein expression of MYC has carefully been described elsewhere to be evenly throughout the tumor (31). To identify tumor cells in more differentiated tumor regions, we co-stained for GFAP (glial fibrillary acidic protein). As exemplarily shown in Fig. 2, all investigated markers could be detected on protein levels in glioblastoma cells. Remarkably, only a small subpopulation of tumor cells showed positive staining for the respective stem cell markers, and these cells were commonly found in small groups in GFAP positive tumor regions. To characterize the proliferative status of glioma cells expressing embryonic stem cell markers, we performed fluorescence double-stainings of OCT4, KLF4 and Nanog with MIB-1/Ki-67, which is a nuclear marker for all active cell cycle phases. Cells with positive staining for OCT4, Nanog or KLF4 did hardly show any MIB-1-signal in the nucleus. Very rare examples of double positive cells are shown in Fig. 3. However, the vast majority of stem cell marker positive cells did not show staining of the proliferation marker MIB-1 (compare also Table III). 
Table III. Evaluation of embryonic stem cell factor co-expression.

\begin{tabular}{|c|c|c|c|c|c|c|c|c|c|c|c|c|}
\hline & \multicolumn{2}{|c|}{ GFAP } & \multicolumn{2}{|c|}{ MIB } & \multicolumn{2}{|c|}{ Sox 2} & \multicolumn{2}{|c|}{ Musashi } & \multicolumn{2}{|c|}{ KLF4 } & \multicolumn{2}{|c|}{ Nanog } \\
\hline & pos & neg & pos & neg & pos & neg & pos & neg & pos & neg & pos & neg \\
\hline \multicolumn{13}{|l|}{ Oct4 } \\
\hline pos & $\bullet$ & + & $\bullet$ & ++ & ++ & $\bullet$ & + & + & + & $\bullet$ & - & + \\
\hline neg & +++ & & ++ & & + & & $\bullet$ & & $\bullet$ & & + & \\
\hline \multicolumn{13}{|c|}{ Nanog } \\
\hline pos & $\bullet$ & + & $\bullet$ & ++ & ++ & • & + & ++ & + & + & & \\
\hline neg & +++ & & ++ & & + & & $\bullet$ & & + & & & \\
\hline \multicolumn{13}{|l|}{ KLF4 } \\
\hline pos & + & + & $\bullet$ & ++ & + & $\bullet$ & $\bullet$ & $\bullet$ & & & & \\
\hline neg & +++ & & ++ & & ++ & & $\bullet$ & & & & & \\
\hline
\end{tabular}

To give a broader impression on the co-expression signature of embryonic stem cell factors we evaluated the immunofluorescence double stainings of four different glioblastomas in a semi-quantitative manner (examples in Figs. 2-4). Although OCT4, KLF4 and Nanog expression was frequently observed in GFAP-positive tumor regions, single expression was more often observed than co-expression. Proliferatively active cells as indicated by the proliferation marker MIB-1/Ki-67 were rarely positive for KLF4, OCT4 and Nanog. Comparing the combinations with the neural stem cell marker Musashi-1, co-expressing cells were almost as frequent as single expressing cells. In combination with SOX2, OCT4 and Nanog were more likely co-stained, whereas KLF4 single-positive cells were more frequent than KLF4-SOX2 double-positive cells. In combinations of Nanog, OCT4 and KLF4 each combination and single expression occurred and was equally represented. However, the total account of embryonic stem cell marker was in the range of 5-10\% respecting intratumoral and intertumoral variances. $\bullet<5 \% ;+>5 \% ;++>10 \%$; $+++>15 \%$. pos, positive; neg, negative.
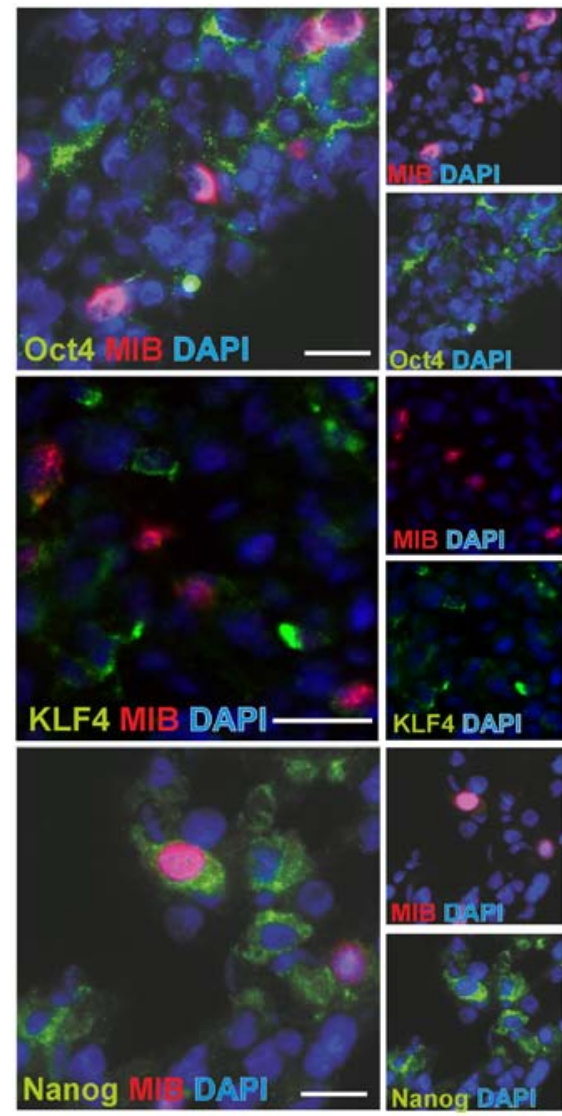

Figure 3. Fluorescence immunostaining of stem cell markers with the proliferation marker MIB in glioblastomas. Glioblastoma cryo-sections were immunostained for MIB-1/Ki-67 (proliferation marker, red) and stem cell markers OCT4, Nanog and KLF4 (green). Stem cell factor expressing cells are rarely positive for the nuclear proliferation marker MIB-1, and vice versa Examples for these stem cell factor expressing and proliferative cells are shown here amongst other, single positive cells. Representatives of 4 glioblastomas, bars indicate $20 \mu \mathrm{m}$.
Co-expression of stem cell markers. To comprehensively analyze the co-expression patterns of embryonic stem cell markers and also compare it to known neural/glioma stem cell markers, we performed fluorescence double-stainings of combinations of the markers OCT4, KLF4 and Nanog with SOX2 and Musashi-1, which are well documented neural/glioma stem cell markers. Representative examples are shown in Fig. 4, and results are summarized in Table III, which also includes the co-staining results with GFAP and MIB-1 in a semi-quantitative evaluation of 4 different glioblastoma samples, respecting the intra- and inter-individual variances. In fact, OCT4, Nanog and KLF4 were partly co-expressed with the other stem cell markers (OCT4, Nanog, KLF4, SOX2 and Musashi-1), but were also found to be expressed in single positive cells. Remarkably, most stem cell marker-expressing cells were also SOX2-positive, but KLF4-negative. When comparing these data to mRNA correlation analysis of investigated stem cell markers (Table IIB), it became apparent that Nanog and OCT4, which showed a positive mRNA correlation to KLF4, respectively, were also frequently co-expressed by the same cell types. Additionally, the missing correlation between KLF4 and SOX2 mRNA expression was underlined by an infrequent KLF4-SOX2 co-staining. Contrasting these findings, Nanog and OCT4, which positively correlated in mRNA expression, were rarely co-expressed. Additionally, a frequently observed co-staining of OCT4 and Nanog with SOX2 was not reflected in a positive correlation of the mRNA expression. Summarized, a heterogeneous picture results which leads to the statement that despite the evidence of a specific role of individual stem cell markers in glioma progression and recurrence, a 'master marker' which is common to all glioblastoma cells with stem cell characteristics, could not be identified. 

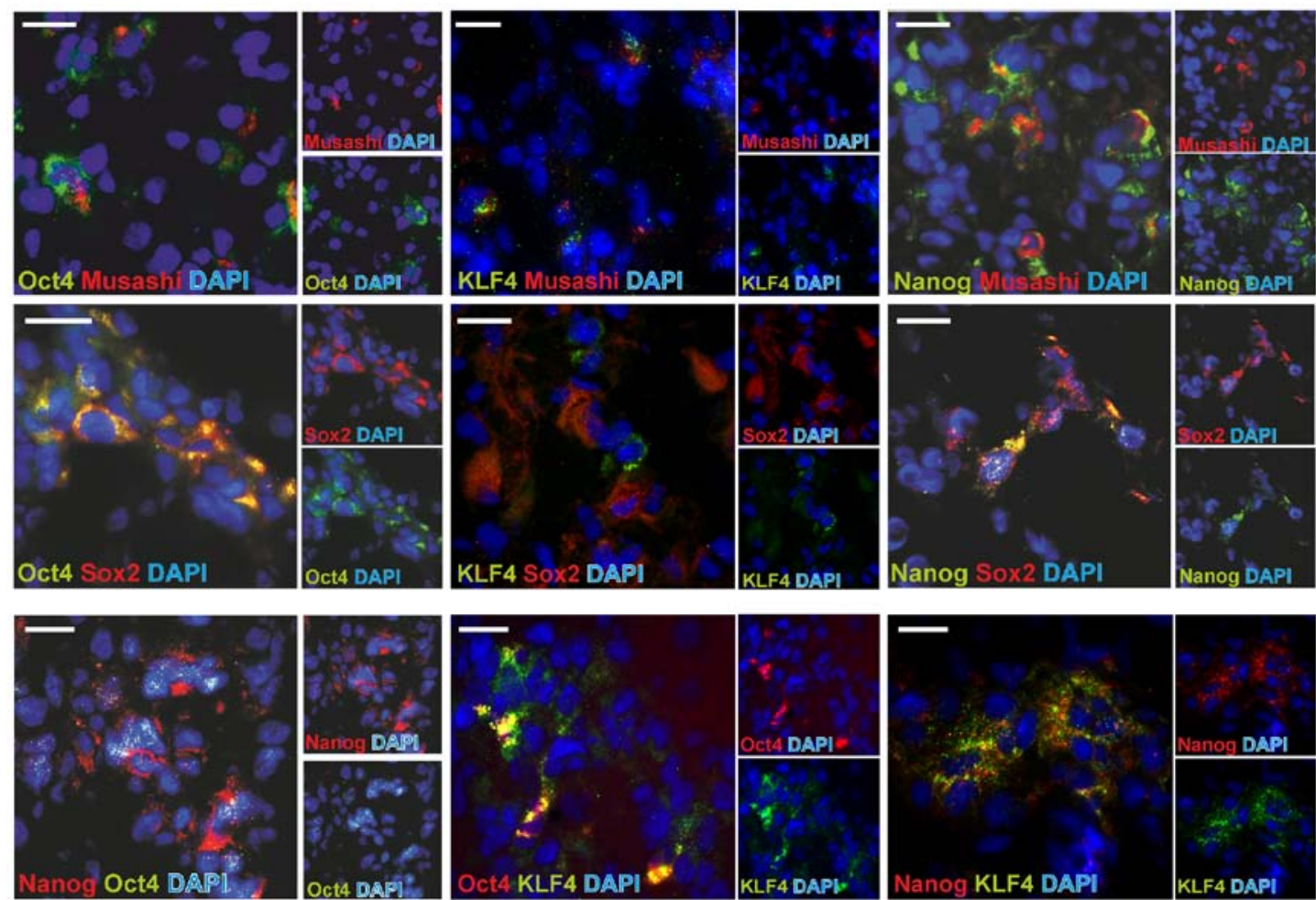

Figure 4. Fluorescence immunostaining of stem cell markers OCT4, Nanog and KLF4 with stem cell markers in glioblastomas (colors as indicated by inscriptions). Glioblastoma cryo-sections were immunostained for OCT4, Nanog and KLF4 in each possible combination, and with Musashi-1 and SOX2. In every staining combination, co-expressing cells and single-positive cells for both antigens were observed. However, OCT4 and Nanog were more likely co-expressed with SOX2, while expression of OCT4, Nanog and KLF4 without SOX2 was rare (compare also Table III). Representatives of 4 glioblastomas, bars indicate $20 \mu \mathrm{m}$

Expression of stem cell factors in glioblastoma short- and long-term cultures and cell lines. For further in vitro investigations, we analyzed the mRNA expression of the stem cell markers OCT4, SOX2, KLF4, MYC, Nanog and Musashi-1 in five commercially available glioma cell lines (A172, U118, U343, U373 and T98G) and two long-term cultures (A764 and A767) as well as four short-term human glioblastoma cultures (14/07, 25/07, 76/12 and 96/14). We found these markers to be expressed on moderate levels in nearly all investigated cell lines as well as in long- and short-term cultures, apart from T98G and 14/07 that rarely expressed Nanog (T98G and 14/07) and SOX2 (T98G), and also comparably low levels of Musashi-1 (T98G; Fig. 5A and B). Interestingly, mRNA expression levels of different stem cell markers showed a surprising range of variation within commercial cell lines and short- and longterm cultures, but within this range cell lines were comparable to long- and short-term cultures. Thus, we chose established glioma cell lines, which are available unlimitedly and therefore solve the problem of limited access to tumor tissue, for further experiments. Experiments mainly focused on the ability to regulate stem cell factors upon extrinsic chemotherapeutic stress and upon intrinsic overexpression of respective stem cell transcription factors.

Regulation of stem cell factors by chemotherapeutics. To investigate the influence of chemotherapeutics on the expression levels of stem cell markers, we chose A172 and T98G as exemplary glioma cell lines because A172 showed moderate to high expression levels of stem cell markers, and T98G showed low expression levels for Nanog and SOX2 (comparable to low expression levels of the short-term cultures 14/07 and $76 / 12$ ). These cell lines were stimulated for 24 or $48 \mathrm{~h}$ with $50 \mu \mathrm{g} / \mathrm{ml}$ camptothecin, or $400 \mu \mathrm{g} / \mathrm{ml}$ temozolomide, the most commonly used chemotherapeutic in glioblastoma treatment; corresponding DMSO concentrations were used for stimulation of solvent controls and used to determine expression changes. For MYC, SOX2 and Musashi-1 we did not observe any significant regulation of the mRNA expression level (data not shown). In contrast, in T98G cells, the expression level of OCT4 was significantly elevated 4.2 -fold ( $\pm 4.3 \mathrm{SD})$ after $48 \mathrm{~h}$ (Fig. 5C). This effect was even more pronounced upon stimulation with camptothecin, but due to high variations not significant. In A172 cells, OCT4 mRNA expression was regulated neither by temozolomide nor by camptothecin. Nanog, which was hardly detectable in unstimulated T98G cells, was found to be increased. However, the proximity to the detection limit has to be kept in mind, thus the data are transparently shaded. In temozolomide-treated A172 cells (48 h), Nanog was significantly but only slightly increased (2.6-fold $\pm 0.7 \mathrm{SD})$, whereas camptothecin yielded no significant effect. Contrasting these slight or little robust expressional changes of OCT4 and Nanog, KLF4 was significantly induced by both temozolomide and camptothecin in T98G and A172 cells. Showing a significant induction after $24 \mathrm{~h}$ already, temozolomide yielded a 17.1 -fold $( \pm 14.0 \mathrm{SD})$, and camptothecin a 40.9 -fold ( $\pm 37.8 \mathrm{SD}$ ) upregulation after $48 \mathrm{~h}$. In A172 cells, an upregulation to 17.7 -fold $( \pm 24.4 \mathrm{SD}$ ) by temozolomide, and to $13.1( \pm 13.2 \mathrm{SD})$ by camptothecin was induced after $48 \mathrm{~h}$. 

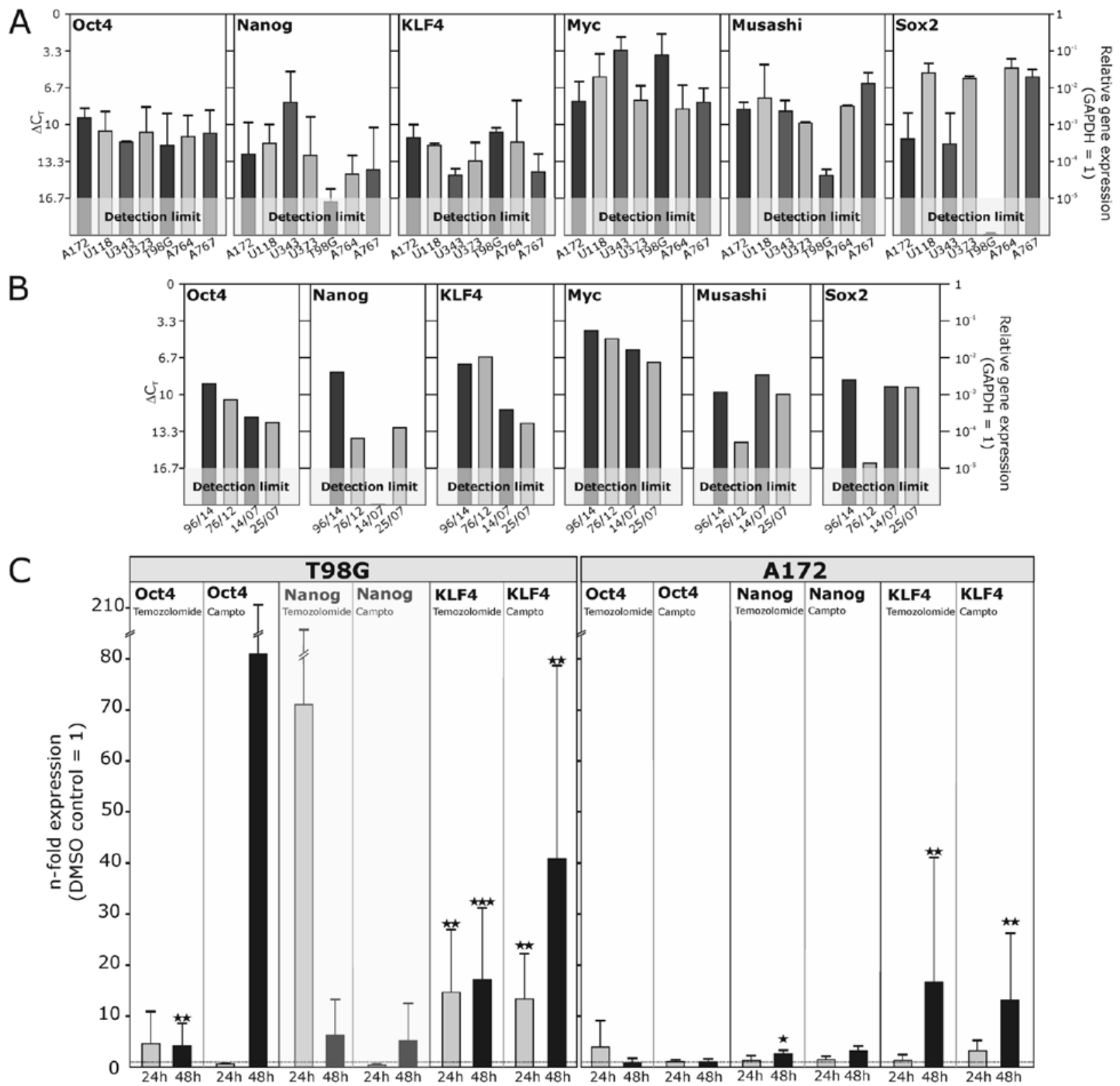

Figure 5. Transcription of stem cell markers in glioblastoma cell lines and short- and long-term primary cultures as well as regulation upon stimulation. (A) The mRNA expression of different commercially available or long-term self-generated glioblastoma cell lines was analyzed by quantitative RT-PCR. While some genes are quite evenly expressed on high, moderate or low levels, like MYC, OCT4 and KLF4, other genes like Nanog, Musashi-1 and SOX2, are more differentially expressed in different cell lines. (B) In short-term glioblastoma primary cultures mRNA expression levels of stem cell markers were comparable to those in commercial cell lines and long-term cultures concerning expression intensity and variability. (C) The glioblastoma cell lines T98G and A172 were stimulated with the cytotoxic drugs temozolomide $(400 \mu \mathrm{g} / \mathrm{ml})$ or camptothecin $(50 \mu \mathrm{g} / \mathrm{ml})$ for 24 and $48 \mathrm{~h}$, respectively, and the regulation of stem cell marker transcription was analyzed by quantitative RT-PCR. Altered gene expression was partly observed for OCT4, Nanog and KLF4, which are displayed as $\mathrm{n}$-fold expression in comparison to the solvent control treated with equal volumes of DMSO. Most robust expression changes were observed for KLF4, which was significantly induced in both cell lines after stimulation with temozolomide or camptothecin. Stimulations were performed in $\mathrm{n}=3$ independent experiments, and a paired Student's t-test was used for statistical analysis.

Summarized, we observed an induction of the embryonic stem cell markers Nanog and KLF4 (and partly OCT4) in glioma cell lines upon chemotherapeutic stress. Surprisingly, this effect is more pronounced in T98G cells, where baseline expression of stem cell markers is comparably low. Among the investigated stem cell markers, KLF4 is most robustly regulated in both glioma cell lines upon extrinsic cytotoxic challenge with camptothecin or temozolomide.

Regulation of stem cell markers by overexpression of transcription factors SOX2, KLF4 or OCT4. Having observed this limited but specific plasticity regarding stem cell marker regulation upon extrinsic challenge, we investigated, if stem cell marker expression might be regulated by intrinsic alteration of stem cell transcription factors SOX2, KLF4 and OCT4. Thus, we performed overexpression experiments with stem cell marker low expressing T98G cells using expression vectors for the respective transcription factors (Fig. 6). Successful transfection was proven either by quantitative RT-PCR (OCT4, 43.6-fold induction $\pm 34.2 \mathrm{SD}$; KLF4, 50.4-fold induction $\pm 36.8 \mathrm{SD}$ ) or in case of SOX2 by immunocytochemistry (insert in Fig. 6). 


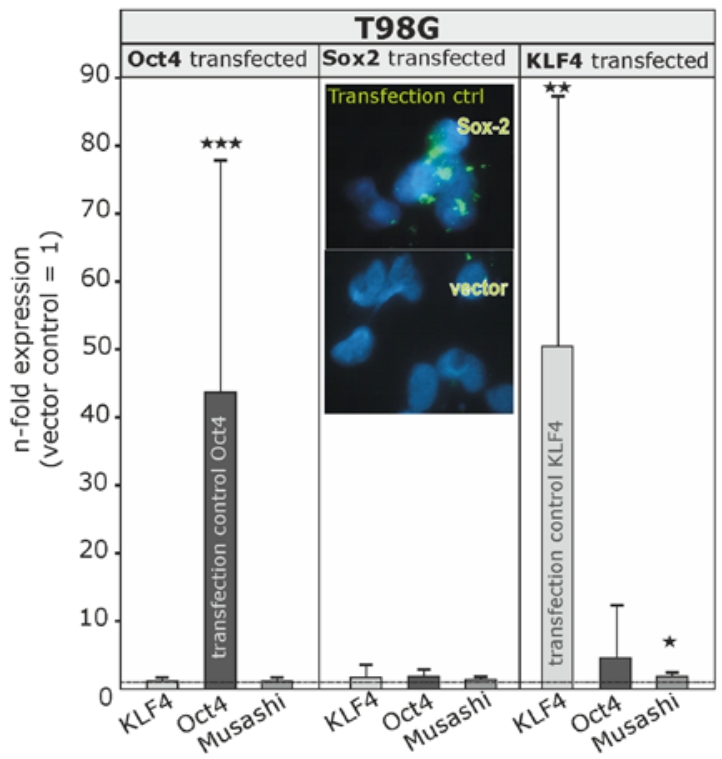

Figure 6. Regulation of stem cell markers upon transfection with the transcription factors OCT4, KLF4 and SOX2. The glioblastoma cell line T98G was transfected with expression vectors for the transcription factors SOX2, KLF4 and OCT4, and transcriptional regulation of stem cell markers (SOX2, KLF4, OCT4, MYC, Musashi-1 and Nanog) in comparison to vector transfected controls was analyzed by quantitative RT-PCR. To control success of transfection, expression was analyzed by quantitative RT-PCR or by immunocytochemistry (SOX2). No changes or very slight changes were observed, as exemplarily displayed here for OCT4 and Musashi-1. Stimulations were performed in $n=3$ independent experiments and a paired Student's t-test was used for statistical analysis.

The overexpression of these transcription factors did not yield a significant regulation (induction or reduction) of other stem cell markers investigated (SOX2, KLF4, OCT4, MYC, Nanog and Musashi-1), apart from Musashi-1, which was only very slightly induced $(1.8$-fold $\pm 0.7 \mathrm{SD})$ upon KLF4 overexpression.

Taken together, the embryonic stem cell transcription factors SOX2, MYC, OCT4, KLF4 and Nanog are expressed in astrocytomas of different malignancy grades as well as in primary and recurrent glioblastomas in a very distinct, probably patient specific expression pattern. Positive correlations between mRNA expression levels of stem cell markers were more pronounced in recurrent glioblastomas, while in situ co-expression occurs in a complex manner, which did not necessarily match the mRNA correlation results. In vitro, stem cell markers were expressed in established cell lines as well as in glioblastoma short- and long-term cultures. The factors were partly regulated by extrinsic chemotherapeutic challenge but not by intrinsic overexpression of the respective transcription factors.

\section{Discussion}

One major concept of tumor development, progression and recurrence is the cancer stem cell hypothesis that postulates the existence of a subpopulation of cancer cells with stem cell properties (like unlimited self-renewal and asymmetric division) within a heterogeneous tumor. Targeting these stem-like cancer cells would be highly desirable, as they are supposed to give rise to recurrences and metastases, e.g. after resection and adjuvant treatment. However, although the existence of tumor cells with stem cell character and the initiation of tumors, recurrences and metastases by tumor cells with stem cell properties have been proven for a multitude of tumors (32-35), to date strategies for clear identification in situ and subsequent systematic targeting of these putatively recurrence-initiating cells in vivo are still missing. In glioma, CD133 was the first marker being identified for glioma stem-like and initiating cells. CD133 is a pentaspan glycoprotein, which is physiologically expressed by neural, hematopoietic and endothelial stem cells $(36,37)$. However, later investigation showed that CD133-negative glioma cells may also have cancer stem cell characteristics such as highly tumorigenic potential (reviewed in ref. 38), and can switch to CD133 expression when they meet the appropriate environment and conditions $(39,40)$. Thus, glioma stem-like cells might be better identified and targeted by their ability to divide unlimitedly and give rise to multifaceted progeny, which can be compared to the pluripotency of embryonic stem cells.

Regarding the fact that as few as four transcription factors can induce pluripotency in differentiated fibroblasts (11), we analyzed mRNA expression of the transcription factors KLF4, OCT4, MYC and SOX2, as well as Nanog, the read out of successful iPSC generation, in astrocytomas of different malignancy grades as well as in matched primary and recurrent glioblastomas by quantitative RT-PCR. We showed, that for most genes transcription was detectable on moderate to high levels in all investigated tumor grades and stages. However, in some individual cases, KLF4, SOX2 and/or Nanog were not detectable. We observed a significant increase in mean mRNA expression for OCT4 in highly malignant astrocytomas (grade III and IV) in comparison to the benign grade I astrocytoma and a slight but significant increase of MYC in grade IV astrocytomas (GBM). For KLF4, an increased transcription was also observed, but due to individual variance statistical robustness could not be proven. An increased expression of SOX2 with increased malignancy of astrocytomas has been reported by us in an earlier study (7). These findings are in line with analysis of Guo et al (41), showing increased protein levels of OCT4 and SOX2 in malignant astrocytomas. They also show increasing expression of Nanog, however, we could not confirm these findings on mRNA level in our sample cohort. Other studies even pointed to higher Nanog levels in astrocytomas and oligodendrogliomas of grade III compared to glioblastomas (42).

Regarding the mean mRNA level in primary and recurrent glioblastomas, we did not observe any significant changes except for MYC, and this mean reduction was comparably mild and with high interindividual variance (ranging from 19 to $217 \%$ ). Interestingly, regarding the individual differences between the matched primary and recurrent samples from the same patients (compare Table I), the cohort could be divided into one group with clear upregulation ( $>2$-fold) of at least one stem cell marker and one group showing predominantly decreased marker transcription. The frequent decrease of stem cell factor expression in recurrences is surprising, as glioma aggressiveness and ability to relapse is often addicted to stem cell properties. Especially the expression of MYC, which was decreased in 11/14 pairs of our matched primaryrecurrent cohort, is known to be significantly increased in 
CD133-positive glioma stem cells. Depletion of CD133 inhibits growth of glioma stem cells, induces apoptosis, reduces ability to glioma sphere formation and inhibits the tumor formation in a murine xenograft model (43). Additionally, high expression levels especially of Nanog, but also of MYC and KLF4 were associated with shorter survival times in a cohort of low and high grade gliomas of different histopathological subtypes, indicating that these embryonic stem cell factors significantly attribute to the clinical outcome of gliomas (8). However, as we for the first time address matched primary and recurrent gliomas, our observations of frequently decreased stem cell factor expression emphasizes a broader view on individualized stem cell factor expression and regulation during progression.

A correlation analysis between stem cell marker expression levels within the investigated malignancy grades and the primary and recurrent glioblastoma cohort revealed distinct positive correlations between individual stem cell marker pairs. Interestingly, in recurrences we observed most frequently significant positive correlations between stem cell marker expressions apart from SOX2.

These correlative findings could, at least in parts, be underlined by co-stainings of the stem cell factors with SOX2, Musashi- 1 and in comparison to each other. Here, we observed on the protein level double stainings for each possible combination but also frequent single expression patterns. Remarkably, especially the significantly positive correlated embryonic stem cell marker pairs like KLF4-OCT4 and KLF4-Nanog were also frequently co-expressed. In contrast to this, less frequent co-expression was observed for the pair Nanog-OCT4, although their expression also positively correlated. Frequent co-expression was observed for SOX2 with the other embryonic stem cell factors, although there was no correlation observed on mRNA level. In earlier studies, a co-expression of Nanog and CD133, and its positive correlation to malignancy grade was reported (44). However, based on our observations, a 'master marker', which uniquely identifies all glioma cells with stem cell characteristics, cannot be defined.

Concerning the intratumoral distribution of embryonic stem cell factors, we observed immunoreactivity for KLF4, OCT4, Nanog and SOX2 in small groups or clusters of cells even within GFAP-positive tumor glioblastoma regions, irrespective of primary or recurrent glioblastoma samples. However, in general embryonic stem cell-like expression signature is more likely observed in poorly differentiated tumors, and the SOX2 expression is known to decrease upon differentiation in vitro $(45,46)$. Concerning co-stainings with the proliferation marker MIB-1/Ki-67, glioma cells expressing the embryonic transcription factor SOX2, like those expressing neural stem cell markers CD133, Nestin and Musashi-1, are rarely actively proliferating (7). Accordingly, we hardly observed any glioma cells expressing OCT4, KLF4 or Nanog with nuclear staining for MIB-1/Ki-67. The majority of stem cell factor positive cells was negative for MIB-1/Ki-67 and also negative for GFAP (though in GFAP-positive regions). These findings hint to an undifferentiated, non-proliferative/resting status of embryonic stem cell factor expressing cells, which is supported by the findings of Holmberg et al (47) concerning SOX2.

In gliomas and other tumors, the expression and regulation of embryonic stem cell markers seems to be critical for the maintenance of tumor stem-like/initiating cells (48-51). The induction of the stem cell factors MYC, KLF4, OCT4, Nanog and SOX2 by hypoxia has been reported for various established cancer cell lines (52). This report also shows an upregulation of OCT4, Nanog and MYC in primary human glioma cells in hypoxic conditions, and an enhanced formation of neurospheres (52). Further investigations demonstrated that signaling of the receptor tyrosine kinase MET/hepatocyte growth factor receptor HGFR could induce the expression of OCT4, KLF4 and Nanog (53). We could additionally show that OCT4, KLF4 and Nanog are at least partly induced upon extrinsic chemotherapeutic treatment. These effects were more prominent in the glioma cell line T98G, which initially showed low expression levels of Nanog, Musashi-1 and SOX2. In this cell line we performed also overexpression experiments with KLF4, OCT4 and SOX2. However, although silencing of OCT4 also provoked downregulation of SOX2 and reduced the tumorigenicity in vitro and in vivo (48), we could not observe any robust regulatory influence of intrinsic overexpression on the expression on any other embryonic or neural stem cell marker. However, recent studies have shown that glioma stem-like cells could be generated by expression of SOX2, OCT4 and Nanog in patient derived glioma cell lines showing neurosphere formation and chemotherapy resistance (54). Taken together, these results implicate that the expression of stem cell factors is altered due to the environmental conditions, so that these conditions, e.g. hypoxia, therapeutic challenge, may influence on the glioma progression.

In conclusion, the embryonic stem cell factors KLF4, OCT4, SOX2, MYC and Nanog are differentially expressed in human astrocytomas and glioblastomas and individually regulated in glioblastoma progression. Their mRNA expression levels partly correlate with each other, especially in recurrent glioblastomas. Staining of stem cell factors in glioblastoma sections occurs solo or in combinations and is restricted to a heterogeneous subpopulation of glioblastoma cells with limited proliferation activity. In correlation analysis and co-stainings, a 'master-marker' defining the complete glioma stem cell subset could not be defined. However, the clear inductions of some stem cell markers and positive correlations especially in recurrent glioblastomas underline the importance of embryonic stem cell factors in glioma progression and recurrence. Extrinsic application of chemotherapeutics but not intrinsic inductions of stem cell transcription factors regulate subsets of stem cell markers. As stem cell markers and transcription factors control self-renewal and tumorigenic potential and therefore glioma progression and recurrence, they may serve as a promising future target for diagnostics and novel therapeutic approaches.

\section{Acknowledgements}

We thank Judith Becker, Martina Burmester, Fereshteh Ebrahim, Jörg Krause, Miriam Lemmers and Brigitte Rehmke for expert technical assistance. Derrick Rossi kindly provided the expression vectors. The present study was supported by grants of the Deutsche Forschungsgemeinschaft (ME758/10-1 and HE3400/51), the State of Schleswig-Holstein (Molecular Imaging in the North MOIN), the popgen 2.0 network (P2N, a grant from the 
German Ministry for Education and Research 01EY1103), and the Family Mehdorn foundation.

\section{References}

1. Ricard D, Idbaih A, Ducray F, Lahutte M, Hoang-Xuan K and Delattre JY: Primary brain tumours in adults. Lancet 379 1984-1996, 2012.

2. Louis DN, Ohgaki H, Wiestler OD, Cavenee WK, Burger PC, Jouvet A, Scheithauer BW and Kleihues P: The 2007 WHO classification of tumours of the central nervous system. Acta Neuropathol 114: 97-109, 2007.

3. Walker C, Baborie A, Crooks D, Wilkins S and Jenkinson MD Biology, genetics and imaging of glial cell tumors. Br J Radio 84 S90-S106, 2011.

4. Bailey P and Cushing $\mathrm{H}$ : A classification of the tumours of the glioma group on a histogenetic basis, with a correlated study of prognosis. Lippincott-Raven, Philadelphia, 1926.

5. Stupp R, Mason WP, van den Bent MJ, Weller M, Fisher B, Taphoorn MJ, Belanger K, Brandes AA, Marosi C, Bogdahn U, et al; European Organisation for Research and Treatment of Cancer Brain Tumor and Radiotherapy Groups; National Cancer Institute of Canada Clinical Trials Group: Radiotherapy plus concomitant and adjuvant temozolomide for glioblastoma. $\mathrm{N}$ Engl J Med 352: 987-996, 2005.

6. Sell S: On the stem cell origin of cancer. Am J Pathol 176: 2584-494, 2010

7. Ma YH, Mentlein R, Knerlich F, Kruse ML, Mehdorn HM and Held-Feindt J: Expression of stem cell markers in human astrocytomas of different WHO grades. J Neurooncol 86: 31-45, 2008

8. Elsir T, Edqvist PH, Carlson J, Ribom D, Bergqvist M, Ekman S Popova SN, Alafuzoff I, Ponten F, Nistér M, Smits A: A study of embryonic stem cell-related proteins in human astrocytomas: Identification of Nanog as a predictor of survival. Int J Cancer 134: 1123-1131, 2014.

9. Dell'Albani P: Stem cell markers in gliomas. Neurochem Res 33: 2407-2415, 2008.

10. Hamerlink P: Cancer stem cells and glioblastoma. In: Glioma Cell Biology. Mentlein R and Sedo A (eds). Springer-Verlag, Wien, New York, pp3-23, 2014

11. Takahashi $\mathrm{K}$ and Yamanaka S: Induction of pluripotent stem cells from mouse embryonic and adult fibroblast cultures by defined factors. Cell 126: 663-676, 2006.

12. Okita K, Ichisaka T and Yamanaka S: Generation of germlinecompetent induced pluripotent stem cells. Nature 448: 313-317, 2007.

13. Okamoto K, Okazawa H, Okuda A, Sakai M, Muramatsu M and Hamada $\mathrm{H}$ : A novel octamer binding transcription factor is differentially expressed in mouse embryonic cells. Cell 60: 461-472, 1990

14. Warren L, Manos PD, Ahfeldt T, Loh YH, Li H, Lau F, Ebina W, Mandal PK, Smith ZD, Meissner A, et al: Highly efficient reprogramming to pluripotency and directed differentiation of human cells with synthetic modified mRNA. Cell Stem Cell 7: 618-630, 2010.

15. Gubbay J, Collignon J, Koopman P, Capel B, Economou A, Münsterberg A, Vivian N, Goodfellow P and Lovell-Badge R: A gene mapping to the sex-determining region of the mouse $Y$ chromosome is a member of a novel family of embryonically expressed genes. Nature 346: 245-250, 1990.

16. Zappone MV, Galli R, Catena R, Meani N, De Biasi S, Mattei E, Tiveron C, Vescovi AL, Lovell-Badge R, Ottolenghi S, et al: Sox 2 regulatory sequences direct expression of a (beta)-geo transgene to telencephalic neural stem cells and precursors of the mouse embryo, revealing regionalization of gene expression in CNS stem cells. Development 127: 2367-2382, 2000.

17. Graham V, Khudyakov J, Ellis P and Pevny L: SOX2 functions to maintain neural progenitor identity. Neuron 39: 749-765, 2003

18. Segre JA, Bauer C and Fuchs E: Klf4 is a transcription factor required for establishing the barrier function of the skin. Nat Genet 22: 356-360, 1999.

19. Chambers I, Colby D, Robertson M, Nichols J, Lee S, Tweedie S and Smith A: Functional expression cloning of Nanog, a pluripotency sustaining factor in embryonic stem cells. Cell 113 643-655, 2003

20. Mitsui K, Tokuzawa $\mathrm{Y}$, Itoh H, Segawa K, Murakami M, Takahashi K, Maruyama M, Maeda M and Yamanaka S: The homeoprotein Nanog is required for maintenance of pluripotency in mouse epiblast and ES cells. Cell 113: 631-642, 2003.
21. Gidekel S, Pizov G, Bergman Y and Pikarsky E: Oct-3/4 is a dose-dependent oncogenic fate determinant. Cancer Cell 4: 361-370, 2003.

22. Peng S, Maihle NJ and Huang Y: Pluripotency factors Lin 28 and Oct4 identify a sub-population of stem cell-like cells in ovarian cancer. Oncogene 29: 2153-2159, 2010.

23. Chiou SH, Wang ML, Chou YT, Chen CJ, Hong CF, Hsieh WJ, Chang HT, Chen YS, Lin TW, Hsu HS, et al: Coexpression of Oct4 and Nanog enhances malignancy in lung adenocarcinoma by inducing cancer stem cell-like properties and epithelial-mesenchymal transdifferentiation. Cancer Res 70: 10433-10444, 2010.

24. Taub R, Kirsch I, Morton C, Lenoir G, Swan D, Tronick S, Aaronson $\mathrm{S}$ and Leder P: Translocation of the c-myc gene into the immunoglobulin heavy chain locus in human Burkitt lymphoma and murine plasmacytoma cells. Proc Natl Acad Sci USA 79: 7837-7841, 1982 .

25. Hemmati HD, Nakano I, Lazareff JA, Masterman-Smith M, Geschwind DH, Bronner-Fraser M and Kornblum HI: Cancerous stem cells can arise from pediatric brain tumors. Proc Natl Acad Sci USA 100: 15178-15183, 2003

26. Wang J, Sakariassen PØ, Tsinkalovsky O, Immervoll H, Bøe SO, Svendsen A, Prestegarden L, Røsland G, Thorsen F, Stuhr L, et al: CD133 negative glioma cells form tumors in nude rats and give rise to CD133 positive cells. Int J Cancer 122: 761-768, 2008.

27. Zhao W, Hisamuddin IM, Nandan MO, Babbin BA, Lamb NE and Yang VW: Identification of Krüppel-like factor 4 as a potential tumor suppressor gene in colorectal cancer. Oncogene 23: 395-402, 2004

28. Jeter CR, Badeaux M, Choy G, Chandra D, Patrawala L, Liu C, Calhoun-Davis T, Zaehres H, Daley GQ and Tang DG: Functional evidence that the self-renewal gene NANOG regulates human tumor development. Stem Cells 27: 993-1005, 2009.

29. Wan F, Herold-Mende C, Campos B, Centner FS, Dictus C, Becker N, Devens F, Mogler C, Felsberg J, Grabe N, et al: Association of stem cell-related markers and survival in astrocytic gliomas. Biomarkers 16: 136-143, 2011.

30. Wang J, Wang H, Li Z, Wu Q, Lathia JD, McLendon RE, Hjelmeland $\mathrm{AB}$ and Rich JN: c-Myc is required for maintenance of glioma cancer stem cells. PLoS One 3: e3769, 2008.

31. Faria MH, Khayat AS, Burbano RR and Rabenhorst SH: c-MYC amplification and expression in astrocytic tumors. Acta Neuropathol 116: 87-95, 2008.

32. Al-Hajj M, Wicha MS, Benito-Hernandez A, Morrison SJ and Clarke MF: Prospective identification of tumorigenic breast cancer cells. Proc Natl Acad Sci USA 100: 3983-3988, 2003.

33. Barker N, Ridgway RA, van Es JH, van de Wetering M, Begthel H, van den Born M, Danenberg E, Clarke AR, Sansom OJ and Clevers H: Crypt stem cells as the cells-of-origin of intestinal cancer. Nature 457: 608-611, 2009.

34. Ignatova TN, Kukekov VG, Laywell ED, Suslov ON, Vrionis FD and Steindler DA: Human cortical glial tumors contain neural stem-like cells expressing astroglial and neuronal markers in vitro. Glia 39: 193-206, 2002.

35. O'Connor ML, Xiang D, Shigdar S, Macdonald J, Li Y, Wang T, Pu C, Wang Z, Qiao L and Duan W: Cancer stem cells: A contentious hypothesis now moving forward. Cancer Lett 344: 180-187, 2014.

36. Galli R, Binda E, Orfanelli U, Cipelletti B, Gritti A, De Vitis S, Fiocco R, Foroni C, Dimeco F and Vescovi A: Isolation and characterization of tumorigenic, stem-like neural precursors from human glioblastoma. Cancer Res 64: 7011-7021, 2004.

37. Singh SK, Clarke ID, Terasaki M, Bonn VE, Hawkins C, Squire J and Dirks PB: Identification of a cancer stem cell in human brain tumors. Cancer Res 63: 5821-5828, 2003.

38. Beier CP and Beier D: CD133 negative cancer stem cells in glioblastoma. Front Biosci (Elite Ed) 3: 701-710, 2011.

39. Nishide K, Nakatani Y, Kiyonari H and Kondo T: Glioblastoma formation from cell population depleted of Prominin1-expressing cells. PLoS One 4: e6869, 2009.

40. Varlakhanova NV, Cotterman RF, deVries WN, Morgan J, Donahue LR, Murray S, Knowles BB and Knoepfler PS: myc maintains embryonic stem cell pluripotency and self-renewal. Differentiation 80: 9-19, 2010.

41. Guo Y, Liu S, Wang P, Zhao S, Wang F, Bing L, Zhang Y, Ling EA, Gao J and Hao A: Expression profile of embryonic stem cell-associated genes Oct4, Sox 2 and Nanog in human gliomas. Histopathology 59: 763-775, 2011.

42. Clement V, Sanchez P, de Tribolet N, Radovanovic I, Ruiz i Altaba A and Altaba R: HEDGEHOG-GLI1 signaling regulates human glioma growth, cancer stem cell self-renewal, and tumorigenicity. Curr Biol 17: 165-172, 2007. 
43. Weina K and Utikal J: SOX2 and cancer: Current research and its implications in the clinic. Clin Transl Med 3: 19, 2014.

44. Niu CS, Li DX, Liu YH, Fu XM, Tang SF and Li J: Expression of NANOG in human gliomas and its relationship with undifferentiated glioma cells. Oncol Rep 26: 593-601, 2011.

45. Ben-Porath I, Thomson MW, Carey VJ, Ge R, Bell GW, Regev A and Weinberg RA: An embryonic stem cell-like gene expression signature in poorly differentiated aggressive human tumors. Nat Genet 40: 499-507, 2008.

46. Hattermann K, Held-Feindt J, Lucius R, Müerköster SS, Penfold ME, Schall TJ and Mentlein R: The chemokine receptor CXCR7 is highly expressed in human glioma cells and mediates antiapoptotic effects. Cancer Res 70: 3299-3308, 2010.

47. Holmberg J, He X, Peredo I, Orrego A, Hesselager G, Ericsson C, Hovatta O, Oba-Shinjo SM, Marie SKN, Nistér M, et al: Activation of neural and pluripotent stem cell signatures correlates with increased malignancy in human glioma. PLoS One 6: e18454, 2011.

48. Ikushima H, Todo T, Ino Y, Takahashi M, Saito N, Miyazawa K and Miyazono K: Glioma-initiating cells retain their tumorigenicity through integration of the Sox axis and Oct4 protein. J Biol Chem 286: 41434-41441, 2011.

49. Kumar SM, Liu S, Lu H, Zhang H, Zhang PJ, Gimotty PA Guerra M, Guo W and Xu X: Acquired cancer stem cell phenotypes through Oct4-mediated dedifferentiation. Oncogene 31: 4898-4911, 2012.
50. Zbinden M, Duquet A, Lorente-Trigos A, Ngwabyt SN, Borges I, Ruiz i Altaba A and Altaba A: NANOG regulates glioma stem cells and is essential in vivo acting in a cross-functional network with GLI1 and p53. EMBO J 29: 2659-2674, 2010.

51. Zhu XY, Wang L, Luan SH, Zhang HS, Huang WT and Wang NH: The PGI-KLF4 pathway regulates self-renewal of glioma stem cells residing in the mesenchymal niches in human gliomas. Neoplasma 61: 401-410, 2014.

52. Mathieu J, Zhang Z, Zhou W, Wang AJ, Heddleston JM, Pinna CMA, Hubaud A, Stadler B, Choi M, Bar M, et al: HIF induces human embryonic stem cell markers in cancer cells. Cancer Res 71: 4640-4652, 2011.

53. Jun HJ, Bronson RT and Charest A: Inhibition of EGFR induces a c-MET-driven stem cell population in glioblastoma. Stem Cells 32: 338-348, 2014.

54. Olmez I, Shen W, McDonald H and Ozpolat B: Dedifferentiation of patient-derived glioblastoma multiforme cell lines results in a cancer stem cell-like state with mitogen-independent growth. J Cell Mol Med 19: 1262-1272, 2015. 